\title{
Importance of perforating vessels in nipple-sparing mastectomy: an anatomical description
}

\author{
This article was published in the following Dove Press journal: \\ Breast Cancer:Targets and Therapy \\ 14 July 2015 \\ Number of times this article has been viewed
}

\author{
Claudio Amanti ${ }^{1}$ \\ Valeria Vitale' \\ Augusto Lombardi' \\ Stefano Maggi' \\ Laura Bersigotti' \\ Gianni Lazzarin' \\ Emiliano Nuccetelli' \\ Camilla Romano' \\ Laura Campanella' \\ Lara Cristiano 2 \\ Alessandra Bartoloni ${ }^{2}$ \\ Giuseppe Argento ${ }^{2}$ \\ 'Breast Surgery Unit, ${ }^{2}$ Radiology \\ Unit, University of Roma, La Sapienza \\ Sant'Andrea Hospital, Rome, Italy
}

Background: Nipple-sparing mastectomy (NSM), understood as an oncologically valid procedure, is relatively new, and is an evolution of traditional mastectomy, particularly in relation to breast-conserving surgery. The anterior perforating branches are responsible for the cutaneous vascularization of the breast skin, and their preservation is a fundamental step to avoid possible postoperative necrosis. Therefore, evaluating the potential complications of cancer-related reconstructive surgical procedures such as NSM, both the distance of the tumoral lesion from the skin and the surgical incision site should be carefully considered. The preferred site of incision corresponds to the inframammary fold or possibly the periareolar area.

Methods: We retrospectively reviewed 113 patients who underwent NSM from January 2005 to October 2012 to evaluate skin complications. The anatomical study was performed by magnetic resonance imaging of the breast.

Results: Only one of the 113 women who had undergone a NSM procedure had total necrosis $(0.9 \%)$ and six patients had partial necrosis $(5.8 \%)$ of the nipple-areola complex.

Keywords: nipple-sparing mastectomy, breast, magnetic resonance imaging, breast perforating vessels, breast anatomy

\section{Introduction}

Nipple-sparing mastectomy (NSM) can be considered as an evolution of traditional mastectomy and represents an oncologically safe procedure, according to the principles of breast conserving surgery.

This technique preserves the entire skin envelope and the nipple-areola complex (NAC), allowing the natural breast shape to be maintained, even after a surgical procedure, in addition to obtaining a better breast reconstruction. ${ }^{1,2} \mathrm{NSM}$ has several indisputable advantages from both an esthetic and a psychological point of view, improving the patient's quality of life and decreasing the feeling of mutilation experienced by women undergoing traditional mastectomy. ${ }^{3}$ In order to reduce the risk of retroareolar recurrence, intraoperative irradiation of the NAC can be performed. ${ }^{4}$

The decision to select a specific incision site depends on several considerations strictly related to the arterial distribution of blood to the mammary gland. As a matter of fact, the elements of surgical anatomy play a decisive role in performing an NSM procedure, when compared with traditional mastectomy. Surgical incision lines along the medial side of the breast are associated with an increased incidence of necrotic events. However, an excessive medial extension of parenchymal dissection may interrupt the blood supply to the skin flaps, and preservation of breast skin vascularization is a prerequisite to reduce the risk of necrosis. One of the main parameters that
Correspondence: Stefano Maggi Breast Surgery Unit, University of Roma, La Sapienza Sant'Andrea Hospital, Via di Grottarossa 1035-1039, 00189 , Rome, Italy

Email stefano.maggi@uniromal.it 
guides the surgical approach to NSM is the tumor-nipple distance, although a preferred type of incision is not yet standardized.

\section{Methods}

The anterior perforating branches of the internal thoracic artery are responsible for the cutaneous vascularization of breast skin. The internal thoracic artery arises from the first portion of the subclavian artery, descends behind the lateral margin of the breast bone, externally to the pleural parietal layer, and ends approximately at the sixth intercostal space; at this level, it splits into two terminal branches, ie, the superior epigastric artery and the musculophrenic artery.

We can identify three perforating branches among the collateral arteries of the internal thoracic artery, ie, the pericardiophrenic artery, the anterior mediastinal artery, and the sternal and anterior intercostal branches. The superior perforating branch emerges from the pectoralis major muscle at the second to third intercostal spaces, an intermediate one, known as "major", at the level of the third to fourth intercostal spaces, while the lower branch emerges in correspondence with the fifth to sixth intercostal spaces. These perforating vessels supply blood to the pectoralis major muscle, to the breast skin envelope, and to the mammary gland.

The vascular pedicle of the Major perforator is composed of: a perforating artery (as shown in Figure 1A), which bifurcates at the parasternal line into a cutaneous ventral branch and a dorsal branch directed to the mammary gland; the anterior cutaneous branch of the intercostal nerve (Figure 2B); and a perforating vein, which is a tributary of the internal thoracic vein. ${ }^{5}$

From anteroposterior projections of the chest wall (Figures 1 and 2), we can recognize: the internal thoracic artery, which runs along the lateral side of the sternum at $1.5-2 \mathrm{~cm}$ from the chondrosternal joint; the sternal branches, originating from the internal thoracic artery, that head first toward the sternum and, after bending medially, end as terminal vessels; and the perforating branches, which emerge
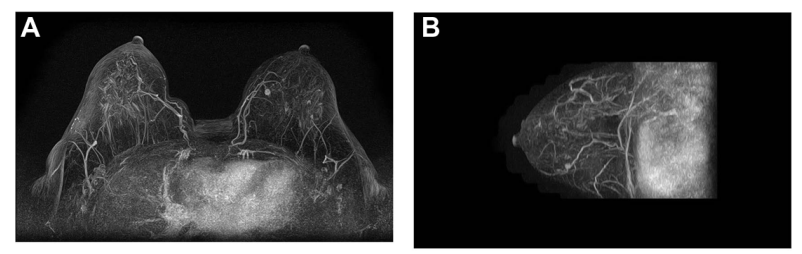

Figure I Maximum intensity projection post-processing three-dimensional angiographic reconstruction images on axial $(\mathbf{A})$ and sagittal $(\mathbf{B})$ view.

Notes: In (A), evidence of enlarged hyperemic anterior perforating arteries are shown on the left breast. In (B) this is well depicted in three-dimensional magnetic resonance angiographic images of the origin of the perforating anterior branches from the internal thoracic arteries.
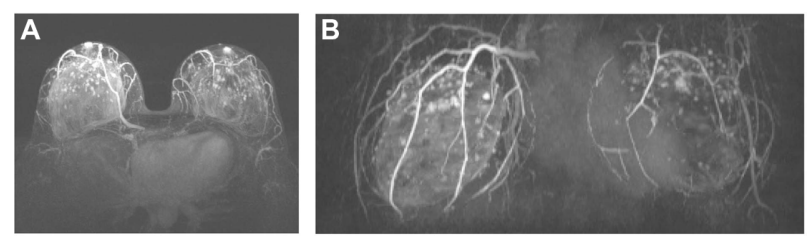

Figure 2 Maximum intensity projection post-processing three-dimensional magnetic resonance angiographic images on axial (A) and coronal (B) views.

Notes: The right breast shows an enlarged hyperemic dorsal branch of the anterior perforating arteries, and (B) shows the origin of cutaneous and nipple-areolar vessels from the anterior perforating branch, known as "Major".

from the internal thoracic artery and are ventrally directed for a few millimeters before bending medially and branching off into two ramifications, ie, a ventral cutaneous branch and a dorsal glandular branch.

Therefore, there exists an extensive network of anastomoses between the lateral thoracic artery and the vessels that originate from the internal mammary (thoracic) artery.

All our preoperative breast examinations are performed with magnetic resonance imaging as part of the internal protocol, employing a dedicated breast coil at $1.5 \mathrm{~T}$ magnetic field intensity (Sonata, Siemens, Washington, DC, USA). The imaging protocol includes turbo spin echo (TSE) T2-weighted axial scans, TSE T1-weighted axial images, sagittal TSE T2-weighted images, T2-weighted axial images, inversion recovery fat-suppressed TIRM T2-weighted axial images under basic conditions (TIRM with both fat and water suppression have been used in presence of silicone implants).

The study is completed with three-dimensional fast low-angle shot T1-weighted fat-saturated sequences with a sequential dynamic study during and after intravenous administration of a paramagnetic contrast agent at a dose of $0.1 \mathrm{mmol} / \mathrm{kg}$, with standard infusion of contrast equal to $2 \mathrm{~mL}$ per second.

After magnetic resonance imaging of the breast, postprocessing subtraction images of the gadolinium-enhanced scans are performed, as well as for the angiographic threedimensional maximum intensity projection reconstructions oriented on coronal, axial, and sagittal views, suitable for displaying the main arterial branches and veins within the breast and their branching from internal thoracic arteries.

Another important observation concerns "wise pattern" and "modified wise pattern" mastectomies. ${ }^{6,7}$ In fact, some authors consider these procedures in the same way as NSM and, also in this case, we can assume the same anatomical considerations as outlined earlier.

The Sant'Andrea Hospital has approved the use of data for this study. 


\section{Results}

We examined a subset of 113 women who had undergone a NSM procedure from January 2005 to October 2012. All the patients are diagnosed with breast cancer $(51 \%$ had multifocal lesions) and the median age was 44 (range: 9-72) years. The literature data referring to NSM-related necrosis events are extremely variable, ranging from $2 \%$ to $20 \%$ and the complete loss of NAC due to necrosis has an incidence in the range of $0 \%-8 \%$, with an average value of $3 \%-4 \%$. The results of our analysis are quite comforting, with total necrosis of the NAC occurring in $0.9 \%$ of cases $(1 / 103)$ and partial necrosis of the NAC in $5.8 \%(6 / 103)$.

\section{Conclusion}

Therefore, in light of our findings, we can underline the importance of the choice of incision site in avoiding postsurgical complications after breast reconstructive surgery, particularly with regard to necrosis of the NAC. To this end, the inframammary fold incision is to be preferred.

\section{Disclosure}

The authors report no conflicts of interest in this work.

\section{References}

1. Rusby JE, Smith BL, Gui GPH. Nipple-sparing mastectomy. Br J Surg. 2010;97:305-316.

2. Moyer HR, Ghazi B, Daniel JR, Gasgarth R, Carlson GW. Nipple-sparing mastectomy - technical aspects and aesthetic outcomes. Ann Plast Surg. 2012;68:446-450.

3. Veronesi U, Stafyla V, Luini A, Veronesi P. Breast cancer: from "maximum tolerable" to "minimum effective" treatment. Front Oncol. 2012; 2:125.

4. Petit JY, Veronesi U, Orecchia R, et al. Nipple sparing mastectomy with nipple areola intraoperative radiotherapy: one thousand and one cases of a five years' experience at the European institute of Oncology of Milan (EIO). Breast Cancer Res Treat. 2009;117:333-338.

5. Chand M, Swan MC, Horlock N, Royle G. Preservation of the lateral thoracic vein in axillary dissection - its role in breast reconstruction using the DIEP flap. Breast. 2009;18:69-70.

6. Santanelli F, Paolini G, Campanale A, Longo B, Adventure C. Modified wise pattern reduction mammoplasty. A new tool for upper quadrantectomies: a preliminary report. Ann Surg Oncol. 2009;16: $1122-1127$.

7. Clough KB, Ihrai T, Oden S, Kaufman G, Massey E, Nos C. Oncoplastic surgery for breast cancer based on tumor location and a quadrant-perquadrant atlas. Br J Surg. 2012;99:1389-1395.

8. Garwood ER, Moore D, Ewing C, et al. Total skin-sparing mastectomy complications and local recurrence rates in 2 cohorts of patients. Ann Surg. 2009;249:26-32.

9. Gieni M, Avram R, Dickson L, et al. Local breast cancer recurrence after mastectomy and immediate breast reconstruction for invasive cancer: a meta-analysis. Breast. 2012;21:230-236.
Breast Cancer: Targets and Therapy

\section{Publish your work in this journal}

Breast Cancer: Targets and Therapy is an international, peerreviewed open access journal focusing on breast cancer research, identification of therapeutic targets and the optimal use of preventative and integrated treatment interventions to achieve improved outcomes, enhanced survival and quality of life for the cancer patient.

\section{Dovepress}

View the full aims and scopes of this journal here. The manuscript management system is completely online and includes a very quick and fair peer-review system, which is all easy to use. Visit http:// www.dovepress.com/testimonials.php to read real quotes from published authors. 